\title{
Solitons in a trapped spin-1 atomic condensate
}

\author{
Wenxian Zhang, ${ }^{1,3}$ Ö. E. Müstecaplığlu, ${ }^{2}$ and L. You ${ }^{3}$ \\ ${ }^{1}$ Ames Laboratory, Iowa State University, Ames, Iowa 50011, USA \\ ${ }^{2}$ Department of Physics, Koç University, Sartyer, Istanbul, 34450, Turkey \\ ${ }^{3}$ School of Physics, Georgia Institute of Technology, Atlanta, Georgia 30332, USA
}

(Received 29 April 2006; published 2 April 2007)

\begin{abstract}
We numerically investigate a particular type of spin soliton inside a trapped atomic spin-1 Bose-Einstein condensate with ferromagnetic interactions. Within the mean-field theory approximation, our study of the solitonic dynamics shows that the solitonic wave function, its center-of-mass motion, and the local spin evolutions are stable and are intimately related to the domain structures studied recently in spin- $1{ }^{87} \mathrm{Rb}$ condensates. We discuss a rotating reference frame wherein the dynamics of the solitonic local spatial spin distribution becomes time independent.
\end{abstract}

DOI: 10.1103/PhysRevA.75.043601

\section{INTRODUCTION}

Solitonic structure is an interesting and common subject in nonlinear science. It has been extensively studied in nonlinear optics [1], fluids [2] and plasmas [3], magnetic films [4], nanotubes [5], and recently in quantum superfluids of atomic Bose-Einstein condensates [6]. In nontechnical terms, the shape of a soliton remains unaltered during propagation due to a balance of the spreading from its dispersion with nonlinear interactions. This feature has been applied with great success in optical-soliton-based advanced communications.

As realized in recent years an atomic Bose-Einstein condensate (BEC) provides a new and powerful tool for the study of nonlinear phenomena. For weak interactions, a condensate is successfully modeled by the Gross-Pitaevskii (GP) equation, or the nonlinear Schrödinger equation [7-12]. The Feshbach resonance in recent years has provided a practical mechanism for adjusting the atomic $s$-wave scattering length through which both the sign and the strength of the atomic interactions become adjustable [13]. Bright matter-wave soliton trains were realized in a ${ }^{7} \mathrm{Li}$ condensate due to its attractive atom-atom interactions, while gray and dark solitons were realized for repulsively interacting condensates through the phase-engineering technique on the condensate wave function $[14,15]$.

Solitons with more than one parameter are often referred to as vector solitons. For instance, vector solitons are created in nonlinear optics if different polarizations or spatial modes of light in an optical fiber are taken as parameters. This could easily be implemented in a spinor condensate as the atomic internal degrees of freedom become available. Many have suggested creating a Manakov soliton in a two-component BEC with attractive self-interactions [16]. Symbiotic solitons have also been investigated in a two-component BEC with repulsive self-interactions accompanied by attractive cross interactions [17]. Domain wall solitons can be created in a trapped two-component condensate even when both self- and cross interactions are repulsive [18]. More generally, selfconsistent collective excitations of an atomic condensate, or higher eigenstates of the Gross-Pitaevskii equation, are also called solitons loosely because of their reasonable stability and resemblance to solitonic states in other properties [19].

With a far-off-resonant optical trap, all hyperfine states of an atom can be confined simultaneously, thus realizing a spinor condensate, as has been reported in many experiments with ${ }^{23} \mathrm{Na}$ and ${ }^{87} \mathrm{Rb}$ atoms [20-28]. The releasing of the spin degree of freedom has led to many novel properties lacking in a single-component or scalar condensate. For instance, the spin-exchange interaction as in a spin-1 condensate, whereby two $\left|F=1, m_{F}=0\right\rangle$ atoms are converted into one $\mid F=1, m_{F}$ $=+1\rangle$ atom, and one $\left|F=1, m_{F}=-1\right\rangle$ atom upon an elastic collision, already well understood [29-31], also helps to support the existence of spinor solitons. In a homogeneous system, exact soliton solutions for an attractively interacting spin- 1 condensate have been recently obtained analytically [32]. Also theoretical investigations have been carried out on magnetic (or spinor) solitons in a ferromagnetically interacting spin-1 condensate $[33,34]$.

In this paper, we focus on the more realistic case of spin solitons inside trapped atomic condensates (of ${ }^{87} \mathrm{Rb}$ ). First we attempt to numerically find solitonic structures through the propagation in imaginary time of the GP equation for a trapped spin- 1 condensate. Then we demonstrate that the dynamics of the found spin solitons is stable and is related to the multidomain structure as studied recently $[35,36]$. Finally, we discuss a rotating reference frame wherein the local spin distribution of the spinor condensate becomes stationary, like a spatially propagating soliton in a moving reference frame.

This paper is organized as follows. In Sec. I we give an introduction and discuss the motivations for our work. The theoretical formulation is based on a model system of a spin-1 condensate as is presented in Sec. II. Section III describes the numerically obtained soliton state, while Sec. IV focuses on the investigation of the solitonic state's dynamics, center-of-mass motion, and local spin distribution. In Sec. V we discuss a rotating reference frame and show that the local spin distribution becomes time independent within this frame. Finally we conclude in Sec. VI.

\section{REVIEW OF AN EFFECTIVE QUASI-ONE- DIMENSIONAL DESCRIPTION}

A gas of interacting spin-1 atoms is described by the following second-quantized Hamiltonian (summation over repeated indices is assumed) [29-31]: 


$$
\begin{aligned}
H= & \int d \vec{r}\left[\Psi_{i}^{\dagger}\left(-\frac{\hbar^{2}}{2 M} \nabla^{2}+V_{\mathrm{ext}}(\vec{r})\right) \Psi_{i}+\frac{c_{0}}{2} \Psi_{i}^{\dagger} \Psi_{j}^{\dagger} \Psi_{j} \Psi_{i}\right. \\
& \left.+\frac{c_{2}}{2} \Psi_{k}^{\dagger} \Psi_{i}^{\dagger}\left(F_{\gamma}\right)_{i j}\left(F_{\gamma}\right)_{k l} \Psi_{j} \Psi_{l}\right],
\end{aligned}
$$

where $\Psi_{j}(\vec{r})\left(\Psi_{j}^{\dagger}\right)$ is the field operator that annihilates (creates) an atom in the $j$ th internal state at location $\vec{r}$, and $j=+, 0,-$ denotes the atomic hyperfine state $\mid F=1, m_{F}$ $=+1,0,-1\rangle$, respectively. $M$ is the mass of each atom, and $V_{\text {ext }}(\vec{r})$ is an internal state-independent trap potential. The terms with coefficients $c_{0}$ and $c_{2}$ of Eq. (1) describe elastic collisions of two spin-1 atoms expressed in terms of the scattering lengths $a_{0}\left(a_{2}\right)$ in the combined symmetric channel of total spin $0(2), c_{0}=4 \pi \hbar^{2}\left(a_{0}+2 a_{2}\right) / 3 M$, and $c_{2}=4 \pi \hbar^{2}\left(a_{2}\right.$ $\left.-a_{0}\right) / 3 M . F_{\gamma=x, y, z}$ are spin- 1 matrices.

The equation of motion for the field operator in the Heisenberg picture is given by

$$
i \hbar \frac{\partial}{\partial t} \Psi_{i}(\vec{r}, t)=\left[\Psi_{i}, H\right] .
$$

Adopting a mean-field theory approach by assuming that the condensate consists of a large number of atoms, we introduce the condensate order parameter or wave function $\Phi_{i}=\left\langle\Psi_{i}\right\rangle$ for the $i$ th component. Neglecting quantum fluctuations we arrive at the coupled Gross-Pitaevskii (GP) equations

$$
\begin{aligned}
& i \hbar \frac{\partial}{\partial t} \Phi_{+}=\left[\mathcal{L}+c_{2}\left(n_{+}+n_{0}-n_{-}\right)\right] \Phi_{+}+c_{2} \Phi_{0}^{2} \Phi_{-}^{*}, \\
& i \hbar \frac{\partial}{\partial t} \Phi_{0}=\left[\mathcal{L}+c_{2}\left(n_{+}+n_{-}\right)\right] \Phi_{0}+2 c_{2} \Phi_{+} \Phi_{-} \Phi_{0}^{*}, \\
& i \hbar \frac{\partial}{\partial t} \Phi_{-}=\left[\mathcal{L}+c_{2}\left(n_{-}+n_{0}-n_{+}\right)\right] \Phi_{-}+c_{2} \Phi_{0}^{2} \Phi_{+}^{*},
\end{aligned}
$$

where $\mathcal{L}=-\left(\hbar^{2} / 2 M\right) \nabla^{2}+V_{\text {ext }}(\vec{r})+c_{0} n, \quad n=\sum_{i} n_{i}$ is the total condensate density, and $n_{i}=\left|\Phi_{i}\right|^{2}$. Another way to derive the above coupled GP equations (3) is to take the variations of the energy functional with respect to the condensate wave function

$$
i \hbar \frac{\partial}{\partial t} \Phi_{i}=\frac{\delta E\left[\Phi_{i}, \Phi_{i}^{*}\right]}{\delta \Phi_{i}^{*}}
$$

where

$$
\begin{aligned}
E\left[\Phi_{i}, \Phi_{i}^{*}\right]= & \int d \vec{r}\left[\Phi_{i}^{*}\left(-\frac{\hbar^{2}}{2 M} \nabla^{2}+V_{\mathrm{ext}}+\frac{1}{2} c_{0} n\right) \Phi_{i}\right. \\
& \left.+\frac{1}{2} c_{2} \Phi_{k}^{*} \Phi_{i}^{*}\left(F_{\gamma}\right)_{i j}\left(F_{\gamma}\right)_{k l} \Phi_{j} \Phi_{l}\right] .
\end{aligned}
$$

Inside a trap with tight radial confinement, the condensate assumes a prolate shape. Several effective one-dimensional (1D) approaches have already been developed [37-42], with the simplest of them assuming a fixed transverse Gaussian profile. Recent studies, however, have indicated that the effective quasi-1D nonpolynomial Schrödinger equation (NPSE) is the more appropriate choice. In fact for most sys- tems of interest, the NPSE represents the most powerful and efficient tool especially in the weakly interacting limit [37-39,42]. In several recent experiments, a single runningwave optical trap is used to confine spin- 1 atomic condensates, a situation well described by the quasi-onedimensional trapping geometry [24-27]. More precisely the external cigar-shaped trap is described by a harmonic trap potential $V_{\mathrm{ext}}=(M / 2)\left(\omega_{\perp}^{2} r_{\perp}^{2}+\omega_{z}^{2} z^{2}\right)$ with $\omega_{x}=\omega_{y}=\omega_{\perp}, r_{\perp}$ $=\sqrt{x^{2}+y^{2}}$, and $\omega_{z} \ll \omega_{\perp}$. The quasi-one-dimensional NPSE description assumes a factorized wave function

$$
\Phi_{i}\left(\vec{r}_{\perp}, z ; t\right)=\sqrt{N} \phi_{\perp}\left(\vec{r}_{\perp} ; \chi(z, t)\right) \phi_{i}(z, t),
$$

into transversal and longitudinal functions [42], which in the case of a spin- 1 condensate are governed, respectively, by

$$
\begin{gathered}
i \hbar \frac{\partial}{\partial t} \phi_{+}=\left[h_{0}+c_{2} N \eta\left(\rho_{+}+\rho_{0}-\rho_{-}\right)\right] \phi_{+}+c_{2} N \eta \phi_{0}^{2} \phi_{-}^{*}, \\
i \hbar \frac{\partial}{\partial t} \phi_{0}=\left[h_{0}+c_{2} N \eta\left(\rho_{+}+\rho_{-}\right)\right] \phi_{0}+2 c_{2} N \eta \phi_{+} \phi_{-} \phi_{0}^{*}, \\
i \hbar \frac{\partial}{\partial t} \phi_{-}=\left[h_{0}+c_{2} N \eta\left(\rho_{-}+\rho_{0}-\rho_{+}\right)\right] \phi_{-}+c_{2} N \eta \phi_{0}^{2} \phi_{+}^{*}, \\
\rho \frac{\partial E_{\perp}}{\partial \chi}+\left(\frac{c_{0} N}{2} \rho^{2}+\frac{c_{2} N}{2} S_{2}\right) \frac{\partial \eta}{\partial \chi}=0 .
\end{gathered}
$$

In the above, $N$ is the total number of condensed atoms. $\phi_{i}$ is the factorized longitudinal envelopes function of the quasione-dimensional wave function, and it depends only on $z$ and t. $\phi_{\perp}$ is the transversal wave function, satisfying $\int d \vec{r}_{\perp}\left|\phi_{\perp}\right|^{2}$ $=1$, and is assumed to be identical for all three spin components. $\chi$ is a variational functional characterizing the width of the transversal wave function. $h_{0}=-\left(\hbar^{2} / 2 M\right)\left(\partial^{2} / \partial z^{2}\right)$ $+V(z)+E_{\perp}+c_{0} N \eta \rho$ with $V(z)=(M / 2) \omega_{z}^{2} z^{2}$, and $E_{\perp}$ is the transverse-mode energy functional,

$$
E_{\perp}(\chi)=\int d \vec{r}_{\perp} \phi_{\perp}^{*}\left(-\frac{\hbar^{2}}{2 M} \nabla_{\perp}^{2}+\frac{1}{2} M \omega_{\perp}^{2} r_{\perp}^{2}\right) \phi_{\perp} .
$$

$\eta(\chi)=\int d \vec{r}_{\perp}\left|\phi_{\perp}\right|^{4}$ is a scaling factor for the nonlinear interaction strength. $\rho(z)=\Sigma_{i}\left|\phi_{i}\right|^{2}$, and $S_{2}$ is independent of $\chi$ and is shown to be given by

$$
\begin{aligned}
S_{2}= & \left|\phi_{+}\right|^{4}+\left|\phi_{-}\right|^{4}+2\left|\phi_{+}\right|^{2}\left|\phi_{0}\right|^{2}+2\left|\phi_{-}\right|^{2}\left|\phi_{0}\right|^{2}-2\left|\phi_{+}\right|^{2}\left|\phi_{-}\right|^{2} \\
& +2 \phi_{0}^{* 2} \phi_{+} \phi_{-}+2 \phi_{+}^{*} \phi_{-}^{*} \phi_{0}^{2} .
\end{aligned}
$$

In obtaining the above relations self-consistently, we have also assumed a weak time and $z$ dependence of the transverse wave function, i.e., $\partial \phi_{\perp} / \partial t \simeq 0$ and $\nabla^{2} \phi_{\perp} \simeq \nabla_{\perp}^{2} \phi_{\perp}$.

For a condensate with a large number of atoms, as in most current experiments, the density distribution of the transversal direction approaches the Thomas-Fermi (TF) limit, $\mu$ $\gg \hbar \omega_{\perp}$; thus we take the TF ansatz for the transverse wave function, 


$$
\phi_{\perp}\left(\vec{r}_{\perp} ; \chi(z, t)\right)= \begin{cases}\sqrt{\frac{2}{\pi}} \frac{1}{\chi} \sqrt{1-\left(\frac{r_{\perp}}{\chi}\right)^{2}}, & r_{\perp} \leqslant \chi, \\ 0, & r_{\perp}>\chi .\end{cases}
$$

The kinetic energy in the transverse direction is therefore neglected under the TF limit, leading to the transverse-mode energy and scaling factor being

$$
\begin{gathered}
E_{\perp}=\frac{\hbar \omega_{\perp}}{6}\left(\frac{\chi^{2}}{a_{\perp}^{2}}\right), \\
\eta=\frac{4}{3 \pi \chi^{2}} .
\end{gathered}
$$

\section{SOLITONS IN A TRAPPED SPIN-1 BOSE CONDENSATE}

The condensate solitons we discuss in this paper are simply self-consistent high-energy eigen-states of the GP equation (3) [19]. Armed with the quasi-1D NPSE approach and the imaginary time propagation method, we devise the following operational procedure for an extensive search of the solitonic states. Numerically we first find the ground state; then we propagate the quasi-1D NPSE Eq. (6) in the imaginary time domain [43], continuously projecting out the ground state at each time step during evolution,

$$
\left|\phi^{(n)}\right\rangle \rightarrow\left|\phi^{(n)}\right\rangle-\left\langle\phi^{(0)} \mid \phi^{(n)}\right\rangle\left|\phi^{(0)}\right\rangle,
$$

hoping for a self-consistent eigensolution to be found. It is well known that different eigensolutions of a nonlinear Hamiltonian may not be orthogonal; furthermore the family of all eigensolutions does not generally constitute an orthornormal and complete basis. Yet our procedure is found to converge at least for the first self-consistent collective excitation. This solitonic state we find is essentially independent of the choice for the transverse mode as being a fixed Gaussian or a fixed TF ansatz [44].

An example of the spin- 1 soliton found in a trapped ${ }^{87} \mathrm{Rb}$ condensate is illustrated in Fig. 1. The scattering lengths used are $a_{0}=101.8 a_{B}$ and $a_{2}=100.4 a_{B}$ with $a_{B}$ being the Bohr radius [45]. In Fig. 1(a) we display the spatial distribution of the local spin average that is defined as

$$
\begin{gathered}
f_{x}(z)=\left\langle F_{x}\right\rangle=\sqrt{2} \operatorname{Re}\left[\phi_{0}\left(\phi_{+}^{*}+\phi_{-}^{*}\right)\right] / \rho, \\
f_{y}(z)=\left\langle F_{y}\right\rangle=-\sqrt{2} \operatorname{Im}\left[\phi_{0}^{*}\left(\phi_{+}-\phi_{-}\right)\right] / \rho, \\
f_{z}(z)=\left\langle F_{z}\right\rangle=\left(\left|\phi_{+}\right|^{2}-\left|\phi_{-}\right|^{2}\right) / \rho,
\end{gathered}
$$

whose magnitude is given by $f(z)=\sqrt{f_{x}^{2}+f_{y}^{2}+f_{z}^{2}}$. We find that the component $f_{y}$, which is displayed perpendicular to the plane, remains very close to zero for the soliton, i.e., the local spins stay almost in the $x-z$ plane. We believe the residual small but nonzero value of $f_{y}$ is due to the numerical precision. Instead of getting rid of these annoying numerical errors, we keep them to study the dynamical stability of the soliton state. Figure 1(a) clearly shows that the local spin

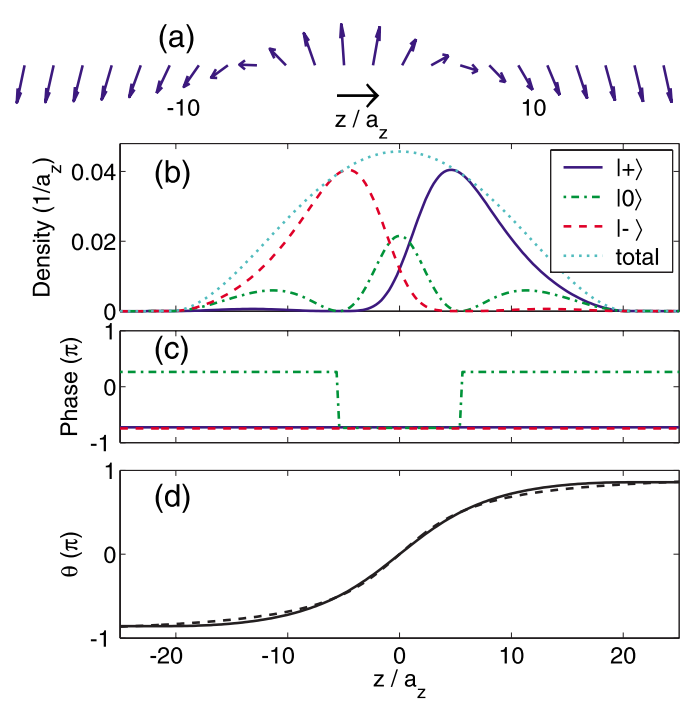

FIG. 1. (Color online) The spatial distribution of the local spin (a), the density (b), and the phase (c) of a soliton state in a trapped spin- $1{ }^{87} \mathrm{Rb}$ condensate. The trap parameters are $\omega_{x}=\omega_{y}=240 \mathrm{~Hz}$, and $\omega_{z}=24 \mathrm{~Hz}$. The total number of atoms in the trap is $N=10^{6}$, and the total magnetization $\left(\mathcal{M}=N_{+}-N_{-}\right)$is zero. The solid curves in (b) and (c) denote the density and the phase for the $|+\rangle$ component, the dashed-dotted curves denote the $|0\rangle$ component, the dashed curves denote the $|-\rangle$ component, and the dotted curve in (b) denotes the total density. The solid line in (d) shows the local spin angle distribution and the dashed line is that for a magnetic kink soliton in a homogeneous system.

performs nearly a complete revolution, or $2 \pi$ around the $y$ axis from one side to the other of the condensate along the quasi-1D axial direction. It is easy to check that the winding number of this solitonic spin distribution is about 1 along the $y$ axis, which shows that the soliton state we find might be related to a quasi-one-dimensional vortex.

We illustrate also the density and the phase distribution in Figs. 1(b) and 1(c). We see a $\pi$ phase shift in the $|0\rangle$ component near the central region where the density of the $|0\rangle$ component diminishes. Also we notice that the $|+\rangle$ and $|-\rangle$ components are symmetric with respect to the trap center at $z=0$. Figure $1(\mathrm{~d})$ shows the angular distribution of the local spin (solid line), $\cos \theta=f_{z} / f$. It is compared to the soliton angular distribution (dashed line) of a magnetic kink soliton given by $\theta=2 \arctan (z / \xi)$ where $\xi=5.4 a_{z}$ and the phase of the $|0\rangle$ component jumps in its neighborhood. The good agreement indicates that the soliton state we find in a trapped spin-1 condensate is closely related to a magnetic kink soliton.

\section{REAL-TIME PROPAGATION OF THE SOLITON IN A TRAP}

Starting from the spinor soliton state found above, we can propagate in real time the quasi-1D NPSE in the trap. This allows us to easily check the dynamic stability of the solitonic structure. In addition, we can check several conservative quantities such as the energy, the total number of atoms $N$, and the total magnetization $\mathcal{M}=N_{+}-N_{-}$to confirm the accu- 


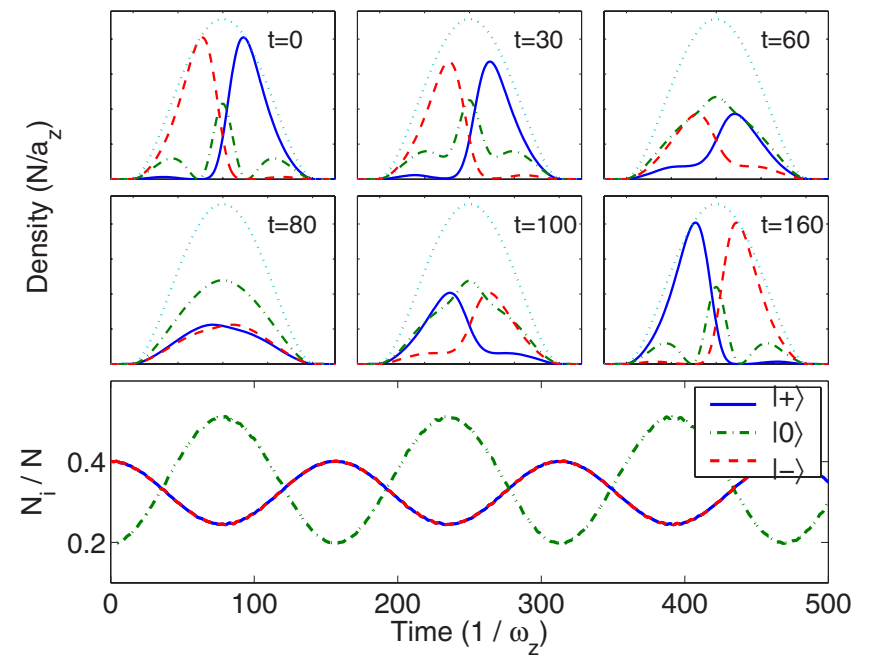

FIG. 2. (Color online) Temporal propagation of the spatial profile of the trapped soliton. The parameters and notations are the same as in Fig. 1. The top and the middle rows display the density distributions of the soliton at different times. The bottom row shows the time dependence of the fractional populations of every spin component.

racy of our numerical integration. During the propagation time $t \in[0,5000]$ (in units of $1 / \omega_{z}$ ), the relative fluctuations of the energy, the number, and the total magnetization are found to be less than $10^{-6}, 10^{-6}$, and $10^{-12}$, respectively.

We show in Fig. 2 the time dependence of the fractional population for each component, clearly periodic in time. The period is found to be $T \sim 313\left(1 / \omega_{z}\right)$, or equivalent to about $2.1 \mathrm{~s}$ in real time units. For a homogeneous condensate this period approaches infinity in the thermal dynamic limit, a topic that is beyond the scope of the present paper and also currently unreachable in experiments. For a finite-sized condensate as discussed here, the period is found to decrease. Figure 2 also shows a typical density distribution for each component at various times during the first half period. We see that the $|+\rangle$ and $|-\rangle$ components tunnel through each other with the assistance of the $|0\rangle$ component and return to their original locations within each period. We note that the $|+\rangle$ and $|-\rangle$ components are immiscible and the tunneling is generally prohibited without the presence of the $|0\rangle$ component. We do not see such an immiscibility here, since the $|0\rangle$ component provides a cohesion for the $|+\rangle$ and the $|-\rangle$ components. This type of tunneling process can also be explained in terms of a mutual precession among the three components as in Ref. [32]. We actually extract the precession angles for both the $|+\rangle$ and $|-\rangle$ components and both are found equal to $\pi$, corresponding to a simple exchange of their positions after the tunneling, i.e., spin up becomes spin down, and vice versa. The interesting feature for a trapped system, distinct from the homogeneous case, is that the tunneling process repeats itself cyclically upon reflections from the trap boundaries.

Figures 3 and 4 display the position and the velocity of the center of mass (c.m.) for each component of the solitonic condensate [46]. Complementary to Fig. 2, Fig. 3 shows the oscillations for the last period of our simulation, which lasts

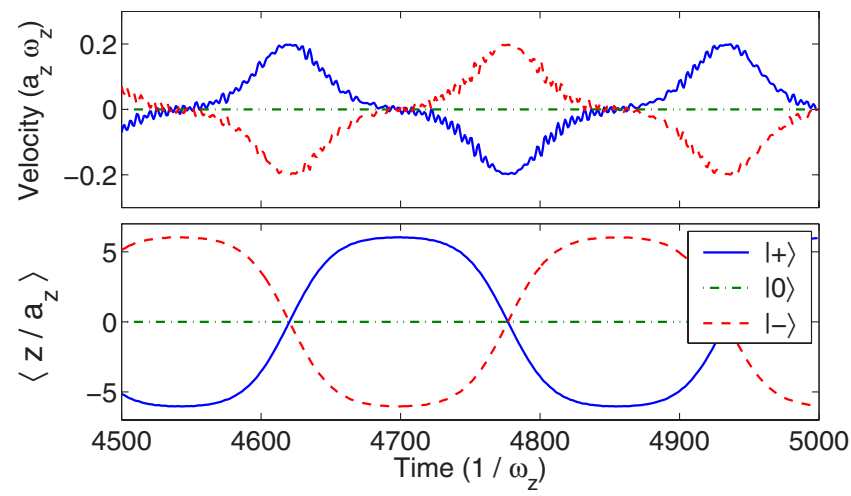

FIG. 3. (Color online) Velocity and position for the center of mass of each soliton component propagating in a trap. The parameters and notations are the same as in Fig. 1. The motion is periodic, and no noticeable distortion occurs after more than 15 periods or about $33 \mathrm{~s}$.

from $t=0$ to $5000\left(1 / \omega_{z}\right)$ with the oscillation period being about $313\left(1 / \omega_{z}\right)$. The velocities of the c.m. for the $|+\rangle$ and the $|-\rangle$ components exhibit many small oscillation structures while that of the $|0\rangle$ component is always very close to zero. We note from Fig. 3 that the position of the c.m. for the $|0\rangle$ component is always zero, but the position for the $|+\rangle$ and the $|-\rangle$ components oscillates back and forth periodically and is symmetrically located on the different sides of that of the $|0\rangle$ component. Figure 4 illustrates an eye-shaped diagram for the phase portrait of the $|+\rangle$ and the $|-\rangle$ components during the first period. The "eye" opens widely with a clear lid, and the $|0\rangle$ component sits at the center. We note that the fluctuations of the velocities for both the $|+\rangle$ and the $|-\rangle$ components are small near the center of the trap and become enhanced around the turning points of trap boundaries.

Now we comment on the issue of dynamical stability of a ferromagnetically interacting spin-1 Bose condensate. Earlier we studied in Ref. [35], the dynamics of a uniformly distributed spin-1 condensate with ferromagnetic interactions is unstable, while in this study we see little distortion of the wave function after an extended real-time propagation even in the presence of initial numerical noise. This clearly indicates the soliton state is dynamically stable. If we compare the soliton

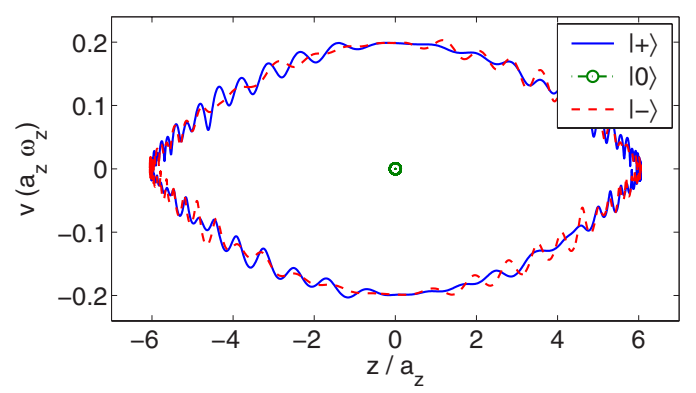

FIG. 4. (Color online) Eye-shaped diagram for the phase portrait of the spinor soliton. The parameters and notations are the same as in Fig. 1. Note that the velocity of the $|0\rangle$ component is very close to zero, and there are many small oscillations for the $|+\rangle$ and $|-\rangle$ components. 


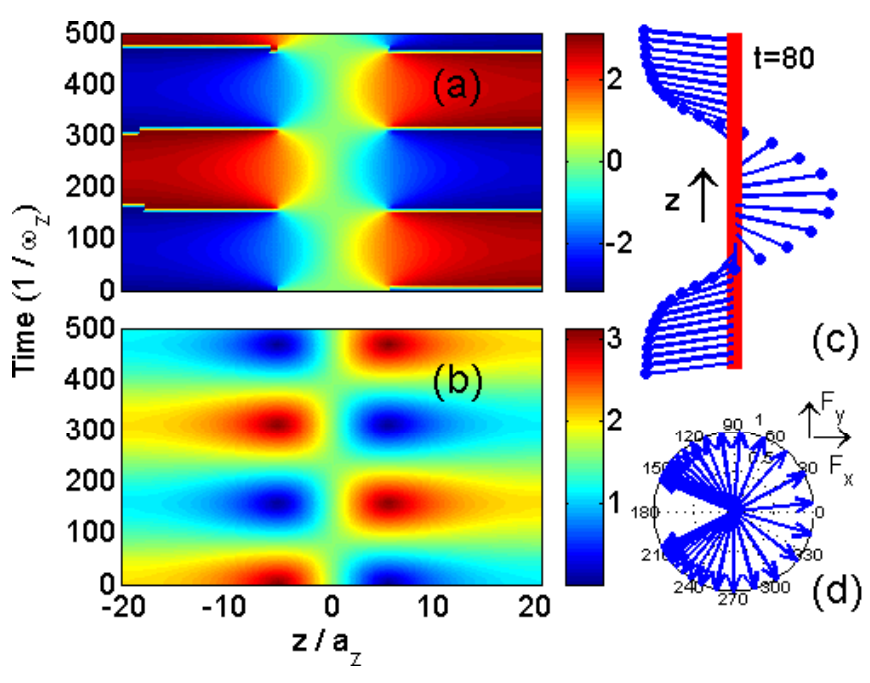

FIG. 5. (Color online) Spatial and temporal distributions of the spin evolution for the spinor condensate soliton. The magnitude of the local spin average is always close to unity due to the ferromagnetic interaction, but the local spin direction evolves with time due to the spin exchange interaction. (a) shows the time dependence of the spatial distribution for the azimuthal spin angle $\varphi$ and (b) that for the polar spin angle $\theta$. (c) shows the typical spatial distribution of the spin average $(t=80)$, and (d) shows the projection of the local spin distributions onto the $x-y$ plane.

state with the final domain structure developed from the homogeneous spin-1 condensate, we see some similarities to their density distributions. These similarities point to a possible connection of the trapped solitonic structure we discuss to the domain structure in the homogeneous case. In more general terms, the domain structure can be interpreted as a certain restricted kind of multiple soliton. Previously, Kasamatsu and Tsubota also noticed these similarities between the domains and the solitons in a system of two-component atomic condensates [47].

We note that the oscillation period is about $2 \mathrm{~s}$, which is of the order of the experimental lifetime of the condensate [24]. Thus, it remains challenging to observe the spin soliton structure we study here. We also observe that the quantum diffusion time of the spin in the ground-state condensate is only a few trap cycles, usually shorter than the period of the soliton oscillations [48]. Furthermore, our investigation focuses on excited-state solitonic structures where the application of the single-spatial-mode approximation may become questionable [48]. A more general treatment that potentially addresses these questions seems completely out of reach for the time being. On the other hand, as we reduced to $N=10^{5}$, still a large number of trapped atoms, the period of the soliton oscillation reduces due to smaller overlap for the $| \pm\rangle$ components to tunnel through. Thus some aspects of these solitonic structures could become observable with the system parameters being tuned to optimize the intended signals before the condensate disappears.

The detailed local spin evolution of the soliton state is further illustrated in Fig. 5. The magnitude of the local spin $f=\sqrt{f_{x}^{2}+f_{y}^{2}+f_{z}^{2}}$ is nearly the same everywhere in space and time. We believe $f$ is exactly the same if a more accurate initial state is used. The spatial distribution of the pointing angles for the local spin, either the azimuthal one $\varphi$ defined by $\tan \varphi=f_{y} / f_{x}$ in Fig. 5(a) or the polar one $\theta$ defined by $\cos \theta=f_{z} / f$ in Fig. 5(b), are strongly periodic. Both angles vary rapidly in the central region and slowly around the boundaries at every moment [the horizontal directions in Figs. 5(a) and 5(b)]. Near the edge of the condensate (the vertical direction), the angle $\varphi$ essentially remains the same at different times (note that $-\pi$ and $\pi$ represent the same spatial direction), while the polar angle $\theta$ changes slightly during each period. $\theta$ changes most rapidly with respect to time at about $z= \pm 6 a_{z}$ where the $|+\rangle$ and the $|-\rangle$ components take their highest densities at $t=0$. In the central region, both angles change moderately with respect to time.

To further clarify the above analysis for the averaged spin directions, we present the $3 \mathrm{D}$ spin distributions at times $t$ $=0$ and $t=80\left(1 / \omega_{z}\right)$ in Figs. 1(a), 5(c), and 5(d), respectively. As stated previously, the local spins have nearly zero projections in the $y$ axis at time $t \simeq 0, T / 2, T, \ldots$, when they are basically lying in the $x-z$ plane and winding around the $y$ axis with a winding number approximately equal to 1 [Fig. 1(a)]. Similarly, $f_{z} \simeq 0$ at times $t \simeq T / 4,3 T / 4,5 T / 4, \ldots$, when the local spins are lying in the $x-y$ plane but winding around the $z$ axis with a similar winding number [Figs. 5(c) and $5(\mathrm{~d})]$. We find that the winding number is a topological quantity that remains conserved during the propagation.

\section{STATIONARY SOLITONS IN A ROTATING REFERENCE FRAME}

A propagating soliton with a constant speed is actually stationary in a special moving frame. A wonderful story about this is that J. S. Russell once followed a water wave soliton for a couple of miles. Such impressive stability during propagation is also manifested for a soliton in a spin-1 condensate. We find that the density of the soliton becomes time independent with the following canonical transformation:

$$
\begin{gathered}
\phi_{a}=\frac{1}{\sqrt{2}}\left(\frac{1}{\sqrt{2}}\left(\phi_{+}+\phi_{-}\right)+\phi_{0}\right), \\
\phi_{b}=\frac{1}{\sqrt{2}}\left(\phi_{+}-\phi_{-}\right), \\
\phi_{c}=\frac{1}{\sqrt{2}}\left(\frac{1}{\sqrt{2}}\left(\phi_{+}+\phi_{-}\right)-\phi_{0}\right) .
\end{gathered}
$$

Figure 6 shows the density distributions of components $|a\rangle,|b\rangle$, and $|c\rangle$, which are all time independent after the transformation. The spatial pattern of the phase for each component is also time independent, i.e., $\theta_{a}$ and $\theta_{c}$ are even functions along the axial coordinate with respect to the center of the condensate, and $\theta_{b}$ has a $\pi$ phase shift at the center of the condensate. We note that this density distribution is similar to that of a Mermin-Ho vortex state of Fig. 1 in Ref. [49], again a potential connection of the solitons and the vortex state.

We can choose a new quantization axis $z^{\prime}$, different from the axial direction of the condensate, such that $\phi_{a}, \phi_{b}$, and 

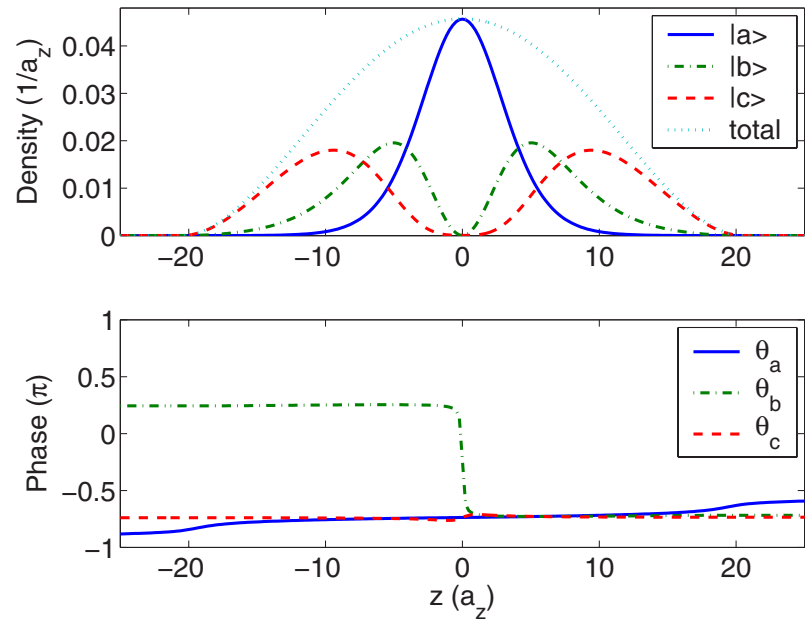

FIG. 6. (Color online) Density and phase distributions of the soliton after the transformation [Eq. (11)]. The density becomes time independent.

$\phi_{c}$ correspond to the wave functions of the $|+\rangle,|0\rangle$, and $|-\rangle$ components in the new coordinate system. We then find that the spatial distribution of $f_{z^{\prime}}=\left|\phi_{a}\right|^{2}-\left|\phi_{c}\right|^{2}$ is time independent (Fig. 6), and the $f_{x^{\prime}}$ and $f_{y^{\prime}}$ rotate with a constant angular velocity around the $z^{\prime}$ axis as shown in Fig. 7. If we go further into the rotating frame defined by

$$
\begin{gathered}
\hat{x}^{\prime \prime}=\hat{x}^{\prime} \cos \Omega t+\hat{y}^{\prime} \sin \Omega t, \\
\hat{y}^{\prime \prime}=-\hat{x}^{\prime} \sin \Omega t+\hat{y}^{\prime} \cos \Omega t, \\
\hat{z}^{\prime \prime}=\hat{z}^{\prime},
\end{gathered}
$$

where $\Omega=2 \pi / T$, we find that the spatial distribution of the spin of the condensate, which is similar to Fig. 1(a), is completely time independent. Thus we show again in the rotating frame (the double primed one) that the appropriate winding number is time independent and remains conserved with change of the reference frame as we confirm previously at the end of Sec. IV.

Further examination shows that the unitary transformation matrix of Eq. (11) is easily decomposed into spin rotations

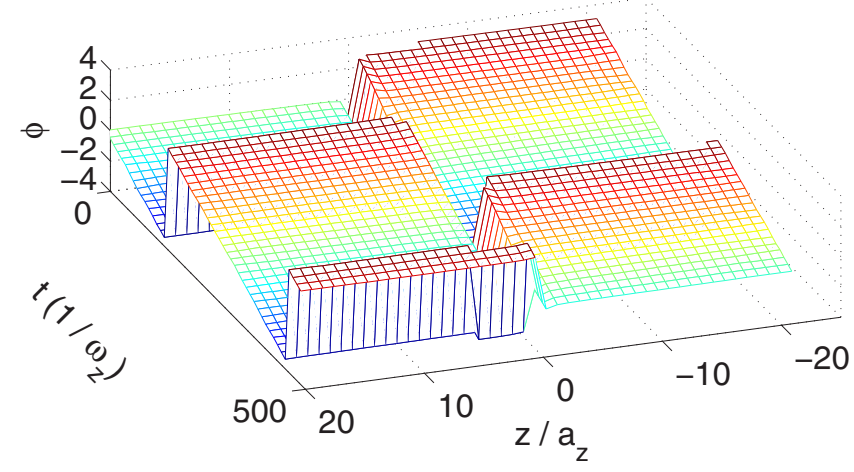

FIG. 7. (Color online) Azimuthal angle of the soliton after the transformation. The spatial pattern of the azimuthal angle is time independent in a rotating reference frame.

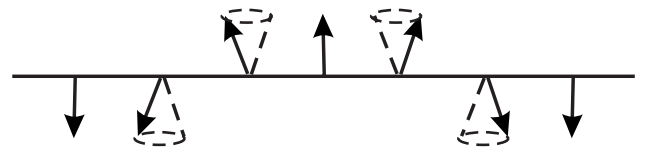

FIG. 8. Schematic diagram of the dynamics of local spins in the new coordinate system.

$$
\begin{aligned}
U & =\left(\begin{array}{ccc}
\frac{1}{2} & \frac{1}{\sqrt{2}} & \frac{1}{2} \\
\frac{1}{\sqrt{2}} & 0 & -\frac{1}{\sqrt{2}} \\
\frac{1}{2} & -\frac{1}{\sqrt{2}} & \frac{1}{2}
\end{array}\right) \\
& =\exp (i \pi) \exp \left(-i F_{y} \frac{\pi}{2}\right) \exp \left(-i F_{z} \pi\right) .
\end{aligned}
$$

The new coordinate $\left(x^{\prime}, y^{\prime}, z^{\prime}\right)$ after the rotation corresponds to the original $(z,-y, x)$. Thus $f_{x^{\prime}}=f_{z}, f_{y^{\prime}}=-f_{y}$, and $f_{z^{\prime}}=f_{x}$. We have checked and confirmed in the original coordinate that $f_{x}$ is indeed time independent, i.e., $f_{z^{\prime}}$ does not change with time (see Fig. 6). We have also found that the time dependence of $\tan ^{-1}\left(-f_{y} / f_{z}\right)=\tan ^{-1}\left(f_{y^{\prime}} / f_{x^{\prime}}\right)=\phi$ is essentially linear as in Fig. 7. Thus we reach the following simplified picture as shown in Fig. 8 in the original coordinates with the given initial conditions as shown in Fig. 1(a). Local spins rotate around the $x$ axis with the same angular frequency $2 \pi / T$. This rotation is constrained so that the $x$ component of the local spin instead of the usual $z$ component is conserved in the discussed soliton state. An amazing aspect of this local spin-precessing dynamics is that the equivalent gyrofrequency is independent of space.

\section{CONCLUSION}

In conclusion, we search numerically for a collectively excited or soliton state in a trapped spin- $1{ }^{87} \mathrm{Rb}$ condensate with ferromagnetic interactions by employing the quasi-onedimensional nonpolynomial Schrödinger equation. We investigate the dynamics of the density, the center-of-mass motion, and the local spin distribution of the solitonic state we find based on a real-time propagation of the NPSE inside the trapped environment. The tunneling process of the $|+\rangle$ and the $|-\rangle$ components with the assistance of the $|0\rangle$ component is explained as mutual precessions among the different components. We further show that the soliton state is dynamically stable and is related to the domain structure observed recently in spin- $1{ }^{87} \mathrm{Rb}$ condensates $[28,35]$. By transforming to a rotating reference frame, we demonstrate that the timedependent dynamics of the local spin of the soliton state becomes time independent. In all of the reported numerical simulations we use $N=10^{6}$ atoms (although unreported simulations were also performed for $N=10^{5}$ as further checks). Within the mean-field theory we find that the results are basically the same except that the period becomes shorter for smaller $N$ due to the reduced overlapped region for the $| \pm\rangle$ components to tunnel through. 


\section{ACKNOWLEDGMENTS}

W.Z. is indebted to Dr. D. L. Zhou for many stimulating discussions, and he is also grateful to Dr. J. Ieda for provid- ing more recent references [32]. This work is supported by NSF and NASA. O.E.M. acknowledges support by Turkish Academy of Sciences TUBA under Young Sceintist Program GEBIP.
[1] G. P. Agrawal, Nonlinear Fiber Optics (Academic Press, Boston, 1989).

[2] P. G. Drazin and R. S. Johnson, Solitons: An Introduction (Cambridge University Press, Cambridge, U.K., 1989).

[3] K. E. Lonngren, Phys. Plasmas 25, 943 (1983).

[4] M. Wu, B. A. Kalinikos, and C. E. Patton, Phys. Rev. Lett. 93, 157207 (2004).

[5] H. Leblond and V. Veerakumar, Phys. Rev. B 70, 134413 (2004).

[6] K. E. Strecker, G. B. Partridge, A. G. Truscott, and R. G. Hulet, Nature (London) 417, 150 (2002).

[7] M. H. Anderson, J. R. Ensher, M. R. Matthews, C. E. Wieman, and E. A. Cornell, Science 269, 198 (1995).

[8] K. B. Davis, M.-O. Mewes, M. R. Andrews, N. J. van Druten, D. S. Durfee, D. M. Kurn, and W. Ketterle, Phys. Rev. Lett. 75, 3969 (1995).

[9] C. C. Bradley, C. A. Sackett, J. J. Tollett, and R. G. Hulet, Phys. Rev. Lett. 75, 1687 (1995); 79, 1170(E) (1997).

[10] C. J. Pethick and H. Smith, Bose-Einstein Condensation in Dilute Gases, (Cambridge University Press, Cambridge, U.K., 2002).

[11] L. Pitaevskii and S. Stringari, Bose-Einstein Condensation (Clarendon Press, Oxford, 2003).

[12] F. Dalfovo, S. Giorgini, L. P. Pitaevskii, and S. Stringari, Rev. Mod. Phys. 71, 463 (1999).

[13] S. L. Cornish, N. R. Claussen, J. L. Roberts, E. A. Cornell, and C. E. Wieman, Phys. Rev. Lett. 85, 1795 (2000).

[14] S. Burger, K. Bongs, S. Dettmer, W. Ertmer, K. Sengstock, A. Sanpera, G. V. Shlyapnikov, and M. Lewenstein, Phys. Rev. Lett. 83, 5198 (1999).

[15] J. Denschlag, J. E. Simsarian, D. L. Feder, C. W. Clark, L. A. Collins, J. Cubizolles, L. Deng, E. W. Hagley, K. Helmerson, W. P. Reinhardt, S. L. Rolston, B. I. Schneider, and W. D. Phillips, Science 287, 97 (2000).

[16] J. Babarro, M. J. Paz-Alonso, H. Michinel, J. R. Salgueiro, and D. N. Olivieri, Phys. Rev. A 71, 043608 (2005).

[17] V. M. Pérez-García and J. B. Beitia, Phys. Rev. A 72, 033620 (2005).

[18] S. Coen and M. Haelterman, Phys. Rev. Lett. 87, 140401 (2001).

[19] D. L. Feder, M. S. Pindzola, L. A. Collins, B. I. Schneider, and C. W. Clark, Phys. Rev. A 62, 053606 (2000).

[20] J. Stenger, S. Inouye, D. M. Stamper-Kurn, H.-J. Miesner, A. P. Chikkatur, and W. Ketterle, Nature (London) 396, 345 (1998).

[21] D. M. Stamper-Kurn, M. R. Andrews, A. P. Chikkatur, S. Inouye, H.-J. Miesner, J. Stenger, and W. Ketterle, Phys. Rev. Lett. 80, 2027 (1998).

[22] D. M. Stamper-Kurn and W. Ketterle, in Spinor Condensates and Light Scattering from Bose-Einstein Condensates, edited by R. Kaiser, C. Westbrook, and F. David, Proceedings of the Les Houches Summer School of Theoretical Physics, LXXII, 1999 (Springer-Verlag, New York, 1999).

[23] M. D. Barrett, J. A. Sauer, and M. S. Chapman, Phys. Rev. Lett. 87, 010404 (2001).

[24] M.-S. Chang, C. D. Hamley, M. D. Barrett, J. A. Sauer, K. M. Fortier, W. Zhang, L. You, and M. S. Chapman, Phys. Rev. Lett. 92, 140403 (2004).

[25] H. Schmaljohann, M. Erhard, J. Kronjäger, M. Kottke, S. van Staa, L. Cacciapuoti, J. J. Arlt, K. Bongs, and K. Sengstock, Phys. Rev. Lett. 92, 040402 (2004).

[26] T. Kuwamoto, K. Araki, T. Eno, and T. Hirano, Phys. Rev. A 69, 063604 (2004).

[27] J. M. Higbie, L. E. Sadler, S. Inouye, A. P. Chikkatur, S. R. Leslie, K. L. Moore, V. Savalli, and D. M. Stamper-Kurn, Phys. Rev. Lett. 95, 050401 (2005).

[28] M.-S. Chang, Q. Qin, W. Zhang, L. You, and M. S. Chapman, Nat. Phys. 1, 111 (2005).

[29] T.-L. Ho, Phys. Rev. Lett. 81, 742 (1998).

[30] T. Ohmi and K. Machida, J. Phys. Soc. Jpn. 67, 1822 (1998).

[31] C. K. Law, H. Pu, and N. P. Bigelow, Phys. Rev. Lett. 81, 5257 (1998).

[32] J. Ieda, T. Miyakawa, and M. Wadati, Phys. Rev. Lett. 93, 194102 (2004); J. Ieda, T. Miyakawa, and M. Wadati, J. Phys. Soc. Jpn. 73, 2996 (2004); M. Wadati and N. Tsuchida, ibid. 75, 014301 (2006); J. Ieda, M. Uchiyama, and M. Wadati, e-print nlin-sys/0603010; M. Uchiyama, J. Ieda, and M. Wadati, J. Phys. Soc. Jpn. 75, 064002 (2006).

[33] Z. W. Xie, W. Zhang, S. T. Chui, and W. M. Liu, Phys. Rev. A 69, 053609 (2004).

[34] Z. D. Li, P. B. He, L. Li, J. Q. Liang, and W. M. Liu, Phys. Rev. A 71, 053611 (2005).

[35] W. Zhang, D. L. Zhou, M.-S. Chang, M. S. Chapman, and L. You, Phys. Rev. Lett. 95, 180403 (2005).

[36] H. Saito and M. Ueda, Phys. Rev. A 72, 023610 (2005).

[37] A. D. Jackson, G. M. Kavoulakis, and C. J. Pethick, Phys. Rev. A 58, 2417 (1998).

[38] L. Salasnich, A. Parola, and L. Reatto, Phys. Rev. A 65, 043614 (2002).

[39] L. Salasnich, Phys. Rev. A 70, 053617 (2004).

[40] M. L. Chiofalo and M. P. Tosi, Phys. Lett. A 268, 406 (2000).

[41] F. Gerbier, Europhys. Lett. 66, 771 (2004).

[42] W. Zhang and L. You, Phys. Rev. A 71, 025603 (2005).

[43] S. Yi, Ö. E. Müstecaplığlu, C. P. Sun, and L. You, Phys. Rev. A 66, 011601(R) (2002); W. Zhang, S. Yi, and L. You, ibid. 70, 043611 (2004).

[44] We find similar solitonic structure with a flatter trap or inside a box but we do not find similar structure in a trapped spin-1 condensate for $c_{2}=0$ or $c_{2}>0$. We note that multicomponent gap solitons of a spin- 1 condensate in an optical lattice have 
been reported by B. J. Dabrowska-wüster, E. A. Ostrovskaya, T. J. Alexander, and Y. S. Kivshar, e-print cond-mat/0612104. [45] E. G. M. van Kempen, S. J. J. M. F. Kokkelmans, D. J. Heinzen, and B. J. Verhaar, Phys. Rev. Lett. 88, 093201 (2002).

[46] G. Huang, M. G. Velarde, and V. A. Makarov, Phys. Rev. A 64, 013617 (2001).
[47] K. Kasamatsu and M. Tsubota, Phys. Rev. Lett. 93, 100402 (2004).

[48] S. Yi, Ö. E. Müstecaplıŏlu, and L. You, Phys. Rev. Lett. 90, 140404 (2003); Phys. Rev. A 68, 013613 (2003).

[49] T. Mizushima, K. Machida, and T. Kita, Phys. Rev. Lett. 89, 030401 (2002). 